\title{
Characterization of pre- and post-treatment pathology after enzyme replacement therapy for pompe disease
}

\author{
Beth L Thurberg ${ }^{1}$, Colleen Lynch Maloney ${ }^{1}$, Charles Vaccaro ${ }^{1}$, Kendra Afonso ${ }^{1}$, \\ Anne Chun-Hui Tsai ${ }^{2}$, Edward $\mathrm{H} \mathrm{Bossen}^{3}$, Priya S Kishnani ${ }^{4,5}$ and Michael O’Callaghan ${ }^{6}$ \\ ${ }^{1}$ Department of Pathology, Genzyme Corporation, Framingham, MA, USA; ${ }^{2}$ Department of Pediatrics, The \\ Children's Hospital of Denver, Denver, CO, USA; ${ }^{3}$ Department of Pathology, Duke University Medical Center, \\ Durham, NC, USA; ${ }^{4}$ Division of Medical Genetics, Duke University Medical Center, Durham, NC, USA; \\ ${ }^{5}$ Department of Pediatrics, Duke University Medical Center, Durham, NC, USA and ${ }^{6}$ Department of \\ Preclinical Biology, Genzyme Corporation, Framingham, MA, USA
}

\begin{abstract}
In Pompe disease, a genetic deficiency of lysosomal acid $\alpha$-glucosidase, glycogen accumulates abnormally in the lysosomes of skeletal, cardiac and smooth muscle, and contributes to clinically progressive and debilitating muscle weakness. The present study involved 8 infantile-onset Pompe patients, treated weekly with $10 \mathrm{mg} / \mathrm{kg}$ of recombinant human acid $\alpha$-glucosidase (rhGAA). Muscle biopsies were obtained at baseline, 12 and 52 weeks post-treatment to establish an indicator of efficacy. Several histologic strategies were employed to characterize changes in pre- and post-treatment samples, including high-resolution light microscopy and digital histomorphometry, electron microscopy, capillary density and fiber type analysis, and confocal microscopy for satellite cell activation analysis. Histomorphometric analysis was performed on muscle samples to assess glycogen depletion in response to enzyme replacement therapy (ERT). The extent of glycogen clearance varied widely among these patient samples, and correlated well with clinical outcome. Low glycogen levels, mild ultrastructural damage, a high proportion of type I fibers, and young age at baseline were all features associated with good histologic response. There was no correlation between capillary density and glycogen clearance, and activated satellite cell levels were shown to be higher in post-treatment biopsies with poor histologic responses. This histopathologic study of infantile Pompe disease provides detailed insight into the cellular progression of the disease and its response to therapy while highlighting a number of methodologies which may be employed to assess regression or progression of the associated pathology. As enzyme replacement therapy becomes more prevalent for the treatment of lysosomal storage diseases, such evaluation of posttreatment pathology will likely become a more common occurrence in the daily practice of pathologists.
\end{abstract}

Laboratory Investigation (2006) 86, 1208-1220. doi:10.1038/labinvest.3700484; published online 30 October 2006

Keywords: acid $\alpha$-glucosidase; enzyme replacement therapy (ERT); glycogen storage disease type II; lysosomal storage disease; pompe disease

Pompe disease (glycogen storage disease type II, acid maltase deficiency) is an autosomal recessive, lysosomal storage disorder in which affected individuals are deficient in the lysosomal enzyme acid $\alpha$-glucosidase (acid maltase). Abnormal lysosomal glycogen accumulates in multiple cell types, particularly in the myocytes of skeletal, cardiac and smooth muscle, and has been detected in fetuses as early as $16-18$ weeks gestation. ${ }^{1,2}$ In the most severe,

Correspondence: Dr BL Thurberg, MD, PhD, Department of Pathology, Genzyme Corporation, One Mountain Road, Framingham, MA 01701-9322, USA.

E-mail: Beth.Thurberg@genzyme.com

Received 7 July 2006; revised and accepted 11 September 2006; published online 30 October 2006 classical infantile form of Pompe disease, macroglossia, cardiomyopathy, hypotonia and respiratory insufficiency dominate with death occurring around the first year of life due to cardiorespiratory failure ${ }^{3-5}$ In the juvenile and adult-onset disease, skeletal muscle weakness predominates. Overall, there is an inverse correlation between disease severity and the level of residual enzyme activity, with the most severely affected infants having no detectable enzyme activity. ${ }^{3}$ Until the recent development of an enzyme replacement, little, other than symptomatic treatment, was available for these patients.

Enzymes such as acid $\alpha$-glucosidase are normally synthesized by the cell, glycosylated and phosphorylated to provide the mannose-6-phosphate 
residue that targets it to the lysosome. Some enzyme is also secreted outside the cell where surface mannose-6-phosphate receptors bind and reinternalize the enzyme and deliver it back to the lysosome. ${ }^{3}$ It is this receptor-mediated reuptake mechanism which allows the targeted delivery of an exogenous enzyme to lysosomes in the setting of a clinical deficiency.

In this 12-month study, we examined the effect of enzyme replacement therapy (ERT) on the pre- and post-treatment pathology of quadriceps biopsies from eight infantile-onset patients, ranging in age from 2.7 to 14.6 months and varying in clinical severity at the start of treatment. The quadriceps muscle was chosen for its early involvement in these patients. We report on the variability of glycogen clearance among the different patient biopsies as evaluated by high-resolution light microscopy (HRLM) and computer-assisted histomorphometry. ${ }^{6}$ We also sought to elucidate the pathophysiologic basis for this variability, employing several histological strategies to examine cellular disease.

Extensive disruption of the contractile apparatus of myocytes has been demonstrated previously in Pompe disease. ${ }^{7}$ Therefore, we examined baseline myocyte ultrastructure by electron microscopy (EM) to determine if the extent of cellular damage correlated with glycogen clearance in post-ERT biopsies. The capacity of muscle to repair and replace severely damaged cells in response to treatment was also investigated. Fluorescent immunohistochemistry and confocal microscopy were employed to examine the activation state of satellite cells, a muscle-specific stem cell population scattered among mature myocytes. Their role in muscle regeneration and repair is well documented. ${ }^{8}$

In both human subjects and animals, blood flow to different muscle groups varies and appears to correlate with muscle fiber type. ${ }^{9,10}$ To assess whether blood flow (and by extension enzyme exposure) correlated with the variable response to ERT, muscle fiber type and capillary density analyses were compared with glycogen clearance. While Pompe disease is generally viewed as a muscular disease, an examination of autopsy tissue from one trial patient provided images which demonstrated that it is in fact a multisystemic disease, and pathologists should be alerted to histologic disease in other organ systems as well.

This study of Pompe pathology presents a spectrum of the histologic disease, its cellular progression and its response to therapy. Before the advent of enzyme replacement therapy, there was little for a pathologist to do beyond diagnosing lysosomal storage diseases on the basis of a biopsy. As we enter an age where lysosomal diseases are not only diagnosed but also treated and monitored, a new field of post-treatment pathology emerges. The work described here utilizes several analytical approaches that may be applied by pathologists in their evaluation of this new class of patient samples.

\section{Materials and methods}

\section{Study Design}

Eight infantile patients (four male patients and four female patients), ranging in age from 2.7 to 14.6 months of age were included in the trial. Clinical disease varied in individual patients from generalized to severe hypotonia at baseline. ${ }^{11}$ Eligibility included a diagnosis of GSDII confirmed by endogenous GAA activity $<1 \%$ of normal in skin fibroblasts. Patients received weekly intravenous infusions of recombinant human acid $\alpha$-glucosidase (rhGAA, $10 \mathrm{mg} / \mathrm{kg}$ ) for an initial 52-week trial period during which biopsies were taken for assessment, followed by enrollment in an extension trial in which patients continued to receive enzyme. Incisional biopsies were obtained from the quadriceps muscle at baseline, 12 and 52 weeks. Biopsy specimens were divided and placed immediately into fresh glutaraldehyde or frozen in OCT (week 52 time point only) before shipment. Glutaraldehyde-fixed and frozen autopsy tissues were also obtained for research purposes from a patient who died after 10 months of ERT. The institutional review boards at all sites approved the open label protocol, and all families gave written informed consent.

\section{High-Resolution Light Microscopy}

Samples were prepared for high-resolution light microscopy (HRLM) according to the procedures of Lynch et al. ${ }^{6}$ To accurately represent the distribution of glycogen across the entire specimen, up to 10 blocks were processed per sample-time point for each patient (Supplementary Figure 1A). One slide from each block was analyzed by computer-assisted histomorphometry (Supplementary Figure 1B); the values obtained were then combined to obtain an average value and standard deviation for each patient-time point (Figure 2).

\section{Computer-Assisted Morphometric Analysis (MetaMorph ${ }^{\circledR}$ )}

One representative field from each slide was photographed with a Nikon DXM1200 digital camera and acquired with the Nikon Act 1 photo image capture software, version 1.12 (Nikon Inc., Instrument Group, Melville, NY, USA). Each field was photographed with the $\times 40$ objective and formatted at a fixed pixel density using Adobe PhotoShop (version 5.5). Each image was opened using the MetaMorph ${ }^{\circledR}$ Imaging Processing and Analysis software (version 4.6; Universal Imaging Corporation) for histomorphometric analysis, as described previously. ${ }^{6}$ MetaMorph ${ }^{\circledR}$ was also utilized to count the number of individual cells at each of the five stages of disease (described in this report) in these same $\times 40$ objective images. The number of cells at each 
disease stage was expressed as a percentage of total cell number and graphed in Microsoft ${ }^{\circledR}$ Excel.

\section{Electron Microscopy}

Epon blocks prepared for HRLM described above were sectioned at $70 \mathrm{~nm}$, mounted onto 200 mesh copper grids, and stained with $1 \%$ aqueous uranyl acetate solution for $20 \mathrm{~min}$ followed by $0.4 \%$ lead citrate/NaOH solution for $30 \mathrm{~s}$. The grids were examined in a Philips EM 300 Electron Microscope (Philips Manufacturing, The Netherlands). Images were collected onto Kodak electron microscope film 4489 and printed on Kodabrome II photographic paper.

\section{Immunofluorescence and Confocal Microscopy}

OCT embedded samples were sectioned at $7 \mu \mathrm{m}$ and stored at $-80^{\circ} \mathrm{C}$. Samples analyzed for co-localization of m-cadherin and myogenin were fixed in $4 \%$ paraformaldehyde $0.2 \mathrm{M} \mathrm{Na} \mathrm{NO}_{4}$ for $60 \mathrm{~min}$. Samples analyzed for co-localization of myogenin and desmin were fixed in acetone at $-80^{\circ} \mathrm{C}$ for $10 \mathrm{~min}$. Samples were rinsed in PBS and incubated for $15 \mathrm{~min}$ in $70 \%$ methanol $/ 0.3 \%$ hydrogen peroxide solution diluted in DI water at RT followed by water rinse. Slides were then incubated for $5 \mathrm{~min}$ in CSK buffer, ${ }^{12}$ rinsed in blocking buffer (PBS,0.5\% bovine albumin, 0.15\% glycine) and incubated at RT with 5\%NGS in blocking buffer for 30-45 min. For m-cadherin/ myogenin co-localization, monoclonal m-cadherin (1:200; BD Biosciences Cat 611101) and rabbit polyclonal myogenin (1:100; Santa Cruz sc-576) were diluted in blocking buffer and $0.1 \%$ Triton. For myogenin/desmin co-localization, monoclonal myogenin (1:50; Dako Cat M3559) and rabbit polyclonal desmin (1:50; Research Diagnostics RDI-PRO 10570) were diluted in blocking buffer only. Samples were incubated with primary antibody for $1.5 \mathrm{~h}$ at $\mathrm{RT}$ and rinsed with blocking buffer. Slides were treated for $30 \mathrm{~min}$ in secondary antibody diluted 1:500 in blocking buffer (AlexaFluor 488-conjugated goat anti-rabbit and goat anti-mouse 546; AlexaFluor 488-conjugated goat anti-mouse and goat anti-rabbit 546; Molecular Probes, Eugene, OR, USA) followed by blocking buffer and PBS rinses. The nuclear counterstain, To-To 3-iodide 642/660, was diluted 1:5000 in PBS and incubated on slides for $15 \mathrm{~min}$ at RT (Molecular Probes, Eugene, OR, USA). Slides were rinsed in PBS and mounted in anti-fade media. Approximately 13-14 nonoverlapping random fields from samples stained for the co-localization of myogenin and m-cadherin were analyzed at $\times 400$ with a confocal laser microscope, Lsm 410 (Carl Zeiss; Germany). Manual counts of data was performed with MetaMorph ${ }^{\mathbb{R}}$ and graphed with Microsoft ${ }^{\mathbb{R}}$ Excel software.

\section{Immunohistochemistry for CD31 and Capillary Density Analysis}

Frozen tissue sections were stained for CD31 (Dako Code M0823, clone JC70A) at a 1:1000 dilution and detected with $\mathrm{DAB}$, following standard immunohistochemical preparation. Digital images of one representative field from each section were acquired with the $\times 20$ objective. All capillaries in each image were counted with MetaMorph ${ }^{\circledR}$ and graphed in Microsoft ${ }^{\circledR}$ Excel.

\section{Fiber Type Staining of Frozen Tissues}

ATPase fiber type staining was performed at $\mathrm{pH} 4.3$, 4.6 and 9.4 on OCT-embedded frozen tissue sections based on published protocols. ${ }^{13,14}$ Sections $(8 \mu \mathrm{m})$ were cut on a cryostat (ThermoShandon, Pittsburgh, PA, USA) and placed on adhesion coated slides. Specimens stained at $\mathrm{pH} 4.3,4.6$ and 9.4 were incubated in either: acetate buffer ( $\mathrm{pH} 4.3$ ) for $5 \mathrm{~min}$; acetate buffer ( $\mathrm{pH}$ 4.6) for $5 \mathrm{~min}$; or calcium barbital buffer ( $\mathrm{pH}$ 9.4) for fifteen minutes. Slides were transferred to an incubating solution, $(0.1 \mathrm{M}$ sodium barbital, $0.18 \mathrm{M}$ calcium chloride, water and adenosine triphosphate, adjusted to a $\mathrm{pH}$ of 9.4 and heated at $37^{\circ} \mathrm{C}$ ) for $20 \mathrm{~min}$. Negative control slides were left in the calcium barbital buffer for the same $20 \mathrm{~min}$. Slides were rinsed in a calcium chloride rinse and then in two cobalt chloride rinses, followed by two to four rinses in distilled water. All slides were then placed into a $1 \%$ ammonium sulfide solution for $3 \mathrm{~min}$ and rinsed two to four times in distilled water. Slides were dehydrated in absolute alcohol to xylene and coverslipped. One representative section from each patient, stained at $\mathrm{pH} 4.3$, was analyzed for percentage of type I fibers using MetaMorph ${ }^{\mathbb{R}}$ analysis.

\section{Results}

\section{Histologic Response to ERT as Assessed by High Resolution Light Microscopy and MetaMorph ${ }^{\circledR}$ Analysis}

Epon-embedded tissues stained with the PAS/ Richardson's combination resulted in HRLM with excellent preservation and staining of glycogen suitable for morphometric analysis. This methodology has been extensively described, and the correlation of PAS staining with glycogen content validated by electron microscopy. ${ }^{6}$ The abnormal glycogen appears purple, and contrasts sharply with the light blue of the surrounding myofibrils (Figure 1), thus permitting digital histomorphometric analysis (Figure 2) of glycogen levels in baseline and posttreatment biopsies. Baseline glycogen accumulation was extensive in all patient biopsies, ranging from 25 to $58 \%$ of total tissue area (Figure 2, white bars). Histologic response to ERT among patient biopsies was highly variable; some demonstrated 

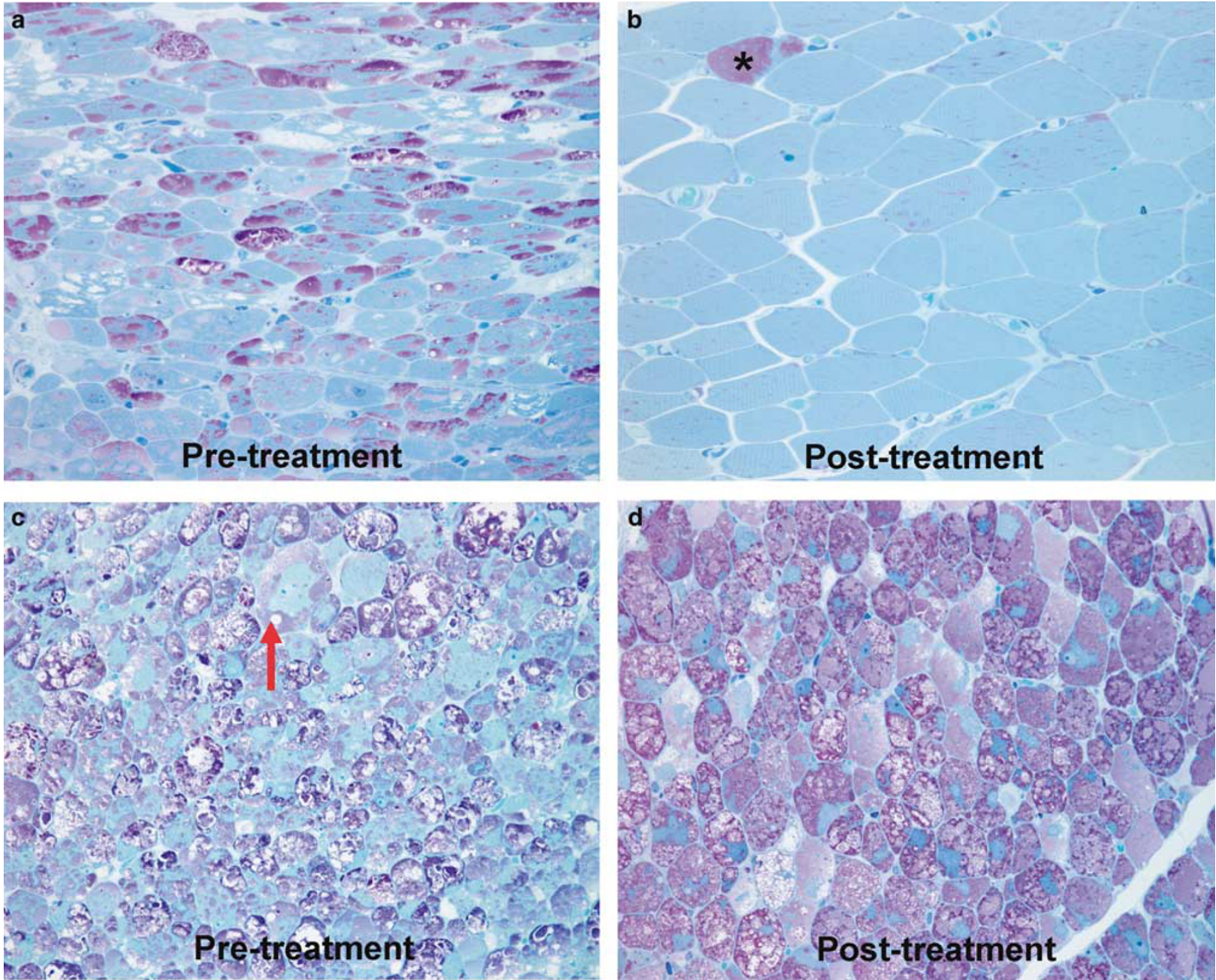

Figure 1 Light microscopic examination of quadriceps biopsies demonstrates that patient biopsies differ in the histologic response to enzyme replacement therapy after 52 weeks of treatment. The glycogen accumulation in patient A at baseline (a) has been cleared in the majority of myocytes after 52 weeks; a rare myocyte appears completely replaced by glycogen and remains unaffected by ERT (asterix, b). (c) Demonstrates the heavy glycogen load present at 3 weeks in patient C; glycogen is distributed at the periphery of many cells (red arrow, c). After 52 weeks of ERT (d), there has been little glycogen clearance, and many myocytes appear completely replaced by glycogen, similar to the appearance of the isolated cell in (b). (HRLM with Richardson's/PAS stain, magnification $\times 400$ ).

dramatic glycogen reduction (Figure 1a and b) while others showed further accumulation (Figure 1c and d). Glycogen levels in patient A, declined from 34 to 4 to $3 \%$ at baseline, week 12 and week 52 , respectively, and similarly for patient $\mathrm{E}$ from 35 to 7 to $1 \%$. These two patients were among the youngest in the group and presented with generalized hypotonia at baseline. The histopathologic changes correlated with improved clinical outcomes; these are extensively described by Kishnani et $a l^{11}$ in a companion clinical publication (Figure 2).

Biopsies from patients $\mathrm{F}$ and $\mathrm{H}$ showed a mixed response. Glycogen levels initially declined from baseline to week 12 (patient F only; baseline sample for patient $\mathrm{H}$ was technically compromised), but subsequently reaccumulated from week 12 to 52 . While these two patients initially showed clinical improvement, particularly patient $\mathrm{F}$ who was able to walk independently for a period of time, both later died.

The remaining four patients (B, C, D and G) had no significant glycogen clearance in response to ERT. Biopsies from patients $B$ and $G$ showed no significant glycogen clearance from baseline to week 12. Both died of disease complications before the 52 week biopsy. Neither patient regained lower limb function and upper body functional improvements were minimal. Biopsies from the remaining two patients, C and D, showed an increase in glycogen from baseline to week 12-52, consistent with their inability to walk, however, improved upper limb strength was reported in both patients. Both subsequently died from complications of the disease after 95 weeks and 71 weeks of treatment, respectively. Of note, patients $\mathrm{C}, \mathrm{D}$ and $\mathrm{G}$ were among the oldest patients in the group and all presented with severe hypotonia at baseline. 


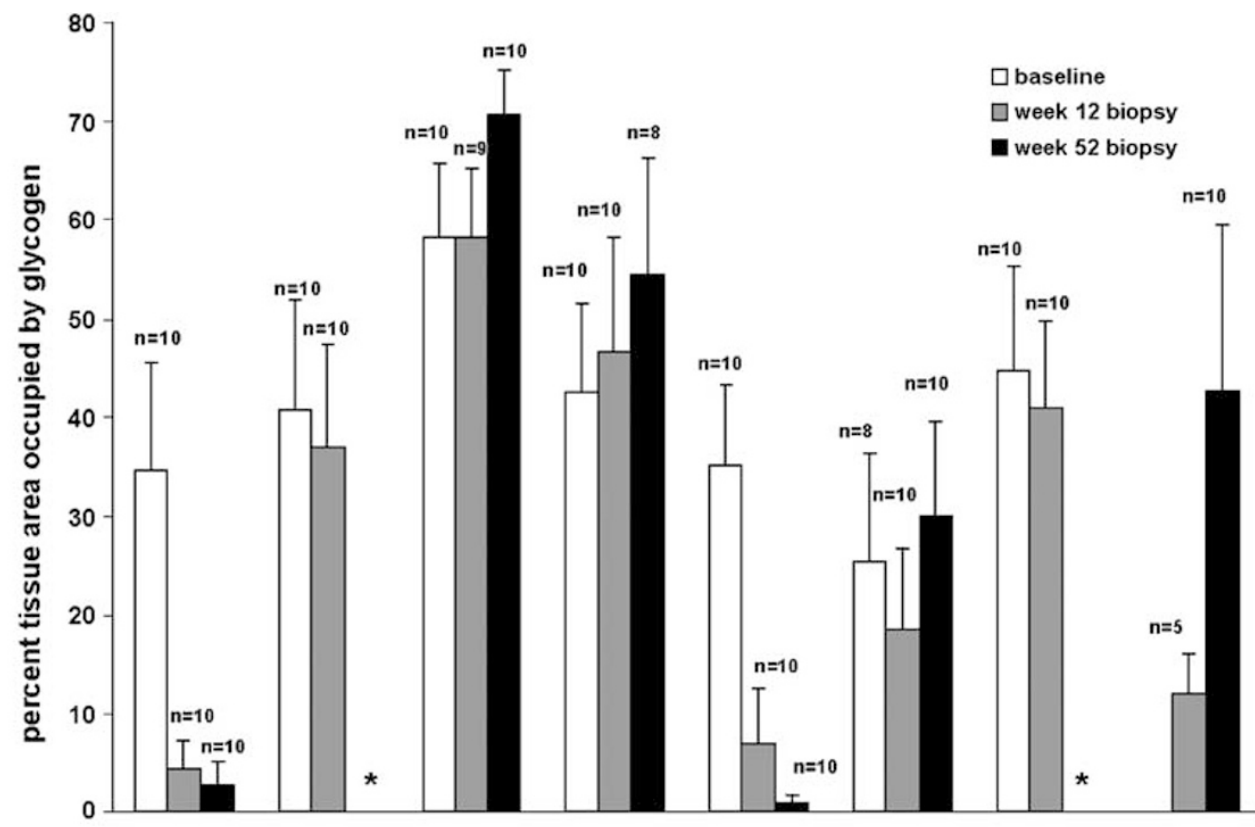

\begin{tabular}{|c|c|c|c|c|c|c|c|c|}
\hline patient & A & B & C & D & $E$ & $\mathrm{~F}$ & G & $\mathrm{H}$ \\
\hline $\begin{array}{c}\text { Age at day } 0 \\
\text { (months) }\end{array}$ & 4.7 & 4.8 & 8.1 & 8.5 & 3.1 & 2.9 & 14.8 & 2.7 \\
\hline $\begin{array}{l}\text { Extent of } \\
\text { baseline } \\
\text { hypotonia }\end{array}$ & $\begin{array}{l}\text { gener } \\
\text {-alized }\end{array}$ & severe & severe & severe & $\begin{array}{l}\text { gener } \\
\text {-alized }\end{array}$ & $\begin{array}{l}\text { gener } \\
\text {-alized }\end{array}$ & severe & $\begin{array}{l}\text { gener } \\
\text {-alized }\end{array}$ \\
\hline $\begin{array}{l}\text { Achieved } \\
\text { upper limb } \\
\text { function }\end{array}$ & yes & $\min$ & yes & yes & yes & yes & $\min$ & yes \\
\hline $\begin{array}{l}\text { Achieved } \\
\text { lower limb } \\
\text { function, } \\
\text { walks }\end{array}$ & yes & no & no & no & yes & yes & no & no \\
\hline $\begin{array}{l}\text { Survival } \\
\text { age dec } \\
\text { wks of tx }\end{array}$ & alive & $\begin{array}{c}\text { dec } \\
14 \mathrm{mo} \\
w k 40\end{array}$ & $\begin{array}{l}\text { dec } \\
34 \mathrm{mo} \\
\text { wk95 }\end{array}$ & $\begin{array}{c}\text { dec } \\
25 \mathrm{mo} \\
w k 71\end{array}$ & alive & $\begin{array}{c}\text { dec } \\
32 \mathrm{mo} \\
w k 87\end{array}$ & $\begin{array}{c}\text { dec } \\
18 m o \\
w k 15\end{array}$ & $\begin{array}{c}\text { dec } \\
23 \mathrm{mo} \\
\text { wk69 }\end{array}$ \\
\hline
\end{tabular}

Figure 2 Metamorph ${ }^{\circledR}$ analysis of glycogen content and correlations with clinical profiles. MetaMorph ${ }^{\circledR}$ analysis of glycogen content was performed on all eight patients at all available timepoints (baseline, week 12 and week 52). 'n' indicates the number of blocks analyzed per biopsy at a given time point. The age of each patient at day 0 of treatment is given on the $x$-axis. Asterisk $\left({ }^{*}\right)$ indicates patients deceased before the 52 week biopsy. Note that baseline samples for patients B and C were delayed until week 3 . No baseline biopsy was available for patient $\mathrm{H}$. The table below summarizes the motor function changes in each patient. A more detailed clinical account of these patients is described elsewhere. ${ }^{11}$ (abbreviations: age $\mathrm{dec}=$ age deceased; wks of $\mathrm{tx}=$ total weeks of ERT).

\section{Assessment of Cellular Damage by EM and Cell Disease Staging}

EM examination of individual myocytes revealed a continuum of ultrastructural disease that could be divided into five stages (Figure 3). These stages could also be identified in corresponding HRLM sections and quantified. Cells with early stage disease contain small, glycogen-filled lysosomes tucked in between intact myofibrils (Stage 1). As 
- Lysosomal glycogen

- Normal mitochondria

- Mild myopathy

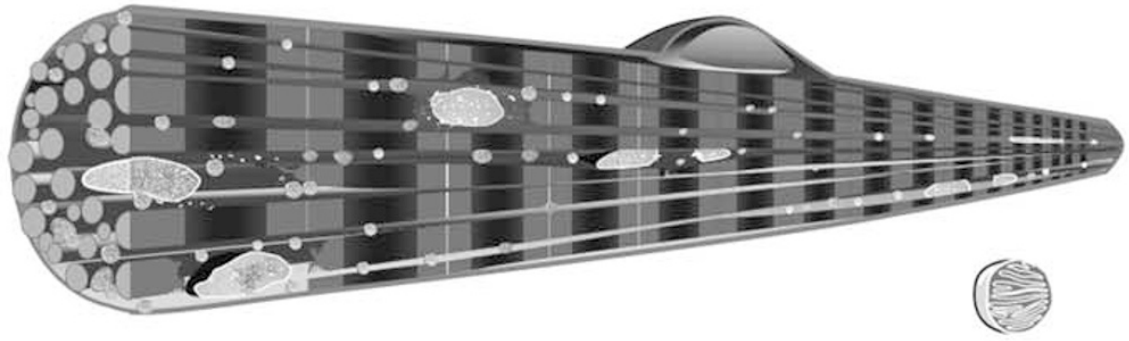

STAGE 1

- Increased lysosomal glycogen

- Patchy cytoplasmic glycogen

- Abnormal mitochondria

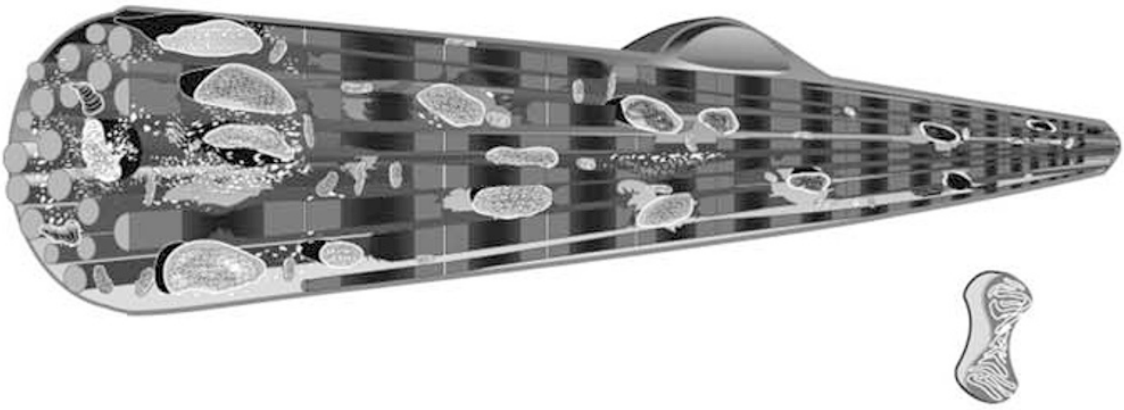

STAGE 2

- Dense lysosomal glycogen

- Increased cytoplasmic glycogen

- Abnormal mitochondria

- Severe myopathy \&

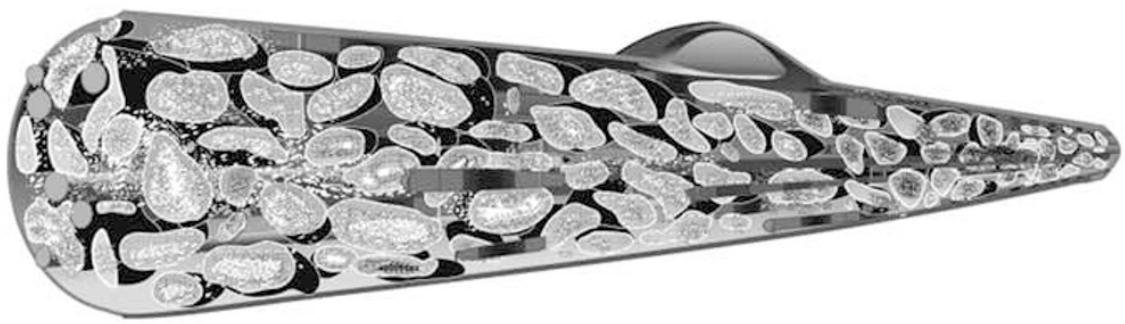

STAGE 3 fibril dissolution

- Decreasing lysosomal glycogen

- Increasing cytoplasmic glycogen

- Scant mitochondria

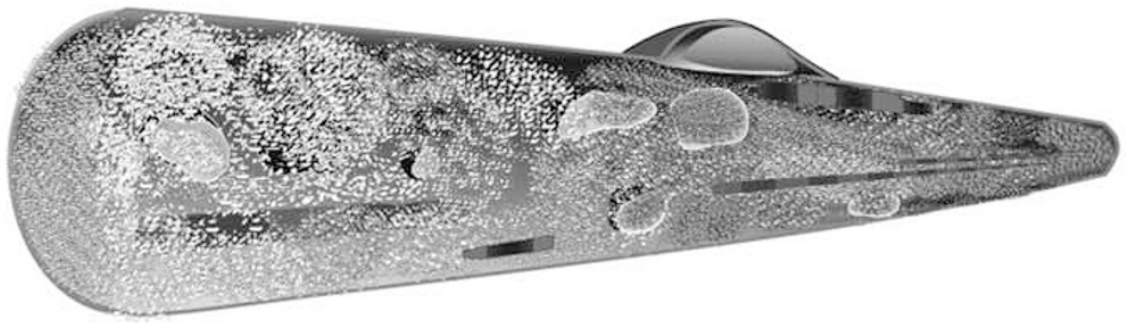

STAGE 4

- Extensive cytoplasmic glycogen

- Cells bloated with edema/water influx

- Complete loss of fibrils \& sarcoplasmic structure

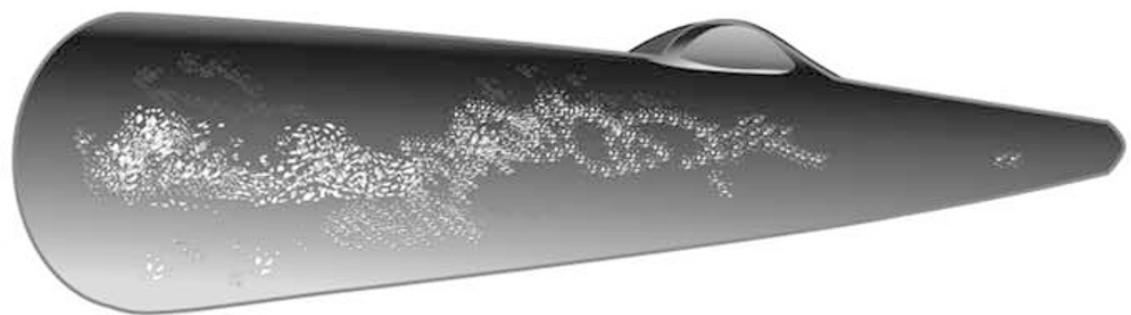

STAGE 5

Figure 3 Cartoon illustration of ultrastructural disease progression in five stages. 
glycogen continues to accumulate, the glycogenfilled lysosomes increase in size and number. In some areas, glycogen has leaked out of the lysosomes and into the cytoplasm and fragmentation of myofibrils is apparent (Stage 2). Stage 3 cells contain numerous, tightly packed glycogen-filled lysosomes. Some lysosomal membranes in these cells have ruptured, allowing more glycogen to float freely in the cytoplasm. Mitochondrial structure is overtly abnormal, and very few myofibril fragments remain. In Stage 4, most glycogen is cytoplasmic and has completely replaced contractile elements of the cell. Stage 5 cells appear bloated, due to the apparent influx of water and resulting dilution or dissolution of glycogen.

Baseline biopsies of patients with good histologic response contained a predominance of myocytes with early Stages 1 and 2 disease (patient A, Figure 4a). Baseline biopsies from poor histologic responders contained a predominance of myocytes with late Stage 3-5 damage, most appearing devoid of intact myofibrils or mitochondria and completely replaced by glycogen. Substrate was often present as a mixture of membrane-bound and cytoplasmic glycogen, and cell debris (patient C, Figure 4b). Figure 4c shows the juxtaposition of a cell with early Stage 2 disease (upper cell) and a cell with late Stage 4 disease (lower cell) at high magnification. The former contains membrane bound glycogen (broken arrow) at the cell periphery with many remaining myofibrils, whereas the latter is completely replaced by cytoplasmic glycogen. (solid arrow) Some myocytes contained intact but abnormally shaped mitochondria (Figure 4d), and others frequently contained tertiary lysosomes filled with cellular debris.

These five stages of cell disease progression could be distinguished at the light level in HRLM sections. Therefore, representative sections were analyzed to quantify the distribution of these five stages. The percentage of each cell stage present in a representative field was quantified for each patient at baseline and at week 52 (when available) and graphed. First, the baseline distributions of seven patients (for whom baseline biopsies were available) were graphed for comparison. Next, five patients with both baseline and week 52 distributions (patients A, C, D, E, F) were graphed individually to assess changes at these two time points.

The baseline distributions (Supplementary Figure 2) show that five of the seven patients had similar distribution patterns with a predominance of Stage 2 cell disease, albeit to differing degrees (A, B, D, E and F). Patients A and E had $>50 \%$ of cells at Stage 2 disease, while $\mathrm{B}, \mathrm{D}$ and $\mathrm{F}$ had $<50 \%$ of cells at Stage 2 disease. The baseline distributions of $C$ and G, by contrast, showed a predominance of Stages 3 and 4 cell disease, respectively. From this graph, one might predict those patients with a predominance of Stage 2 cell disease to respond well to ERT, whereas those patients with baseline distributions shifted to the right ( $C$ and $G$ ), signifying more severe disease, might be predicted to do poorly.

The post-treatment distributions of patients A and E (Supplementary Figure 2) demonstrate a marked shift to the left with a predominance of stage zero (normal) cell disease after 52 weeks of enzyme replacement; these patients continue to do well clinically. By contrast, the distributions of patients $\mathrm{D}$ and $\mathrm{F}$ demonstrated a gradual shift to the right at week 52, signifying progressive disease. The distribution of patient $\mathrm{C}$ showed a marked shift to the right, with a rapid increase in late cell disease stage. Patients C, D and F died of progressive disease.

\section{Satellite Cell Activation}

Frozen, week 52 biopsies were examined for the presence of quiescent and activated satellite cells (no frozen baseline samples were available from this study). Satellite cells could occasionally be identified in epon sections by electron microscopy (Figure 4e). However, in frozen samples, double fluorescent labeling (Figure 4f) with antibodies to M-cadherin (red cell membrane) and myogenin (green nuclei) permitted the quantification of all satellite cells, expressed relative to all cell nuclei (blue). Mcadherin is expressed on all satellite cells, quiescent and activated; myogenin is expressed only in activated satellite cells. The percentage of all nuclei associated with M-cadherin positive cell membranes ranged from 31 to $48 \%$ (Supplementary Table 1; Supplementary Figure 3A) in 52 week biopsies. The percentage of myogenin positive (activated) satellite cells varied from 1.2 to $5.6 \%$ among the patient samples (Supplementary Table 1; Supplementary Figure 3B). Patients A and E (both good histologic responders) had the lowest percentage of activated satellite cells (1.2 and $2.1 \%$, respectively), while D had the highest percentage of activated cells $(5.6 \%)$. Rare, activated satellite cells could be observed fusing with one another, exhibiting increased desmin expression, as they matured into multinucleated myocytes. Week 52 samples were unavailable for patients B and G.

\section{Capillary Density and Fiber Type Analyses}

Frozen, week 52 biopsy samples were stained for CD31 to identify capillaries. Capillary density ranged from 102 to 219 capillaries per $\times 20$ objective field (Supplementary Figure 3C). There was no obvious correlation between capillary density and glycogen clearance. However, MetaMorph ${ }^{\circledR}$ analysis of frozen sections stained for fiber type (ATPase $\mathrm{pH}$ 4.3) demonstrated a trend toward higher type I fiber content in good histologic responders. Biopsies from patients $\mathrm{A}$ and $\mathrm{E}$, the best responders, contained 44 and $31 \%$ type I fibers, respectively (Supplementary Figure 3D). Patient F, who was also an initial responder with the ability to walk, demonstrated a 

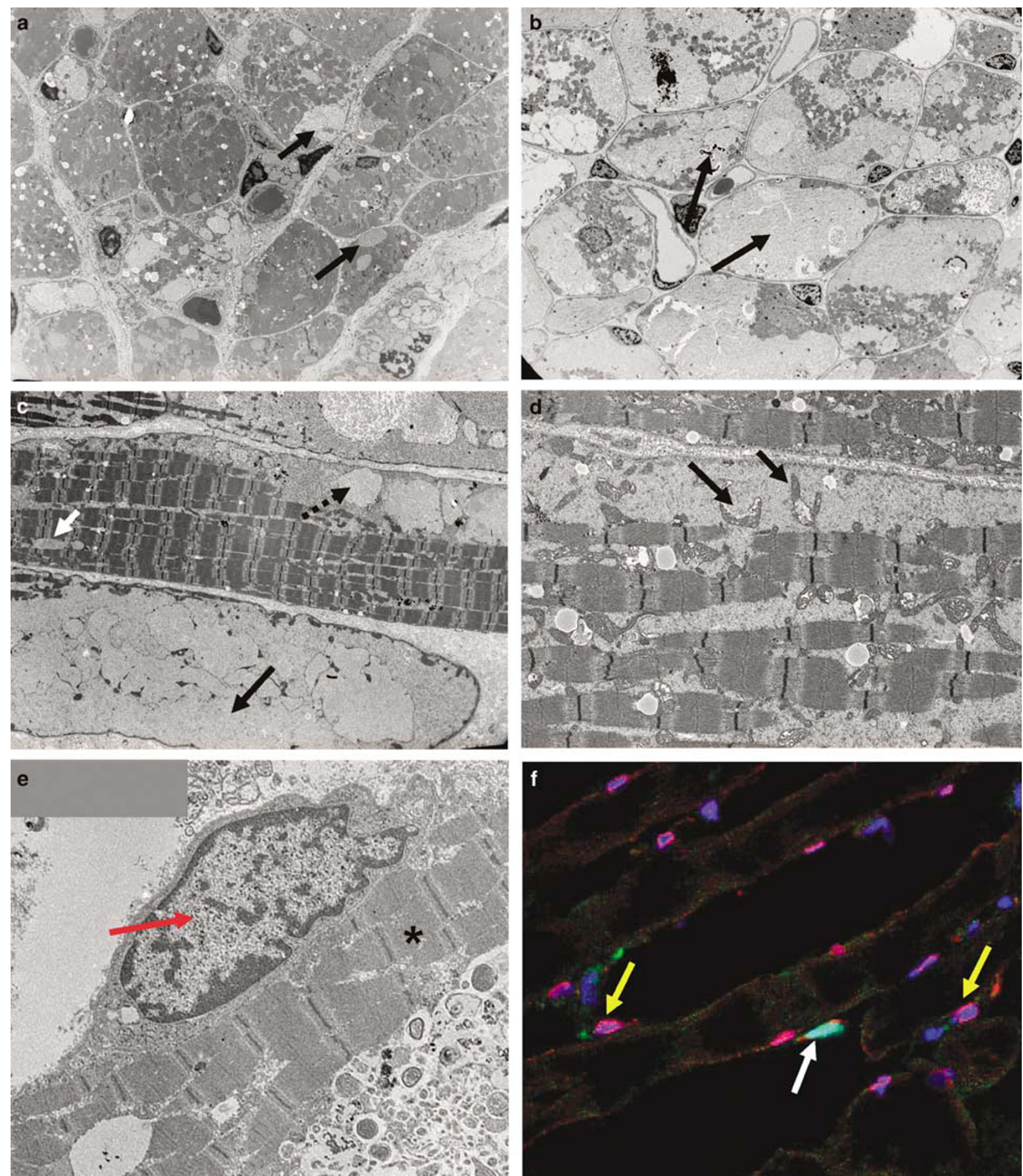

Figure 4 Ultrastructural disease correlates with response to ERT. (a) Myocytes with early Stage 1 or 2 ultrastructural disease predominate in the baseline biopsy of patient A, a good histologic responder. Most cells are full of intact myofibrils with lysosomal glycogen tucked in between $(4500 \times)$. (b) Myocytes with late Stage 3 or 4 ultrastructural disease predominate in the baseline biopsy of patient C, a poor histologic responder. Most cells lack myofibrillar structure and are filled with glycogen lakes and debris $(\times 4500)$. (c) High-power view of the variation in cell damage $(\times 6500)$. The upper cell with early Stage 2 disease consists of many intact contractile elements with fraying at the fibril ends and peripheral, membrane-bound glycogen (broken arrow). The lower cell with Stage 4 disease has been completely replaced by cytoplasmic glycogen (solid arrow). Ruptured lysosomal membranes float freely in the lake of glycogen. No contractile elements or mitochondria remain. (d) High-power view of a cell with early Stage 2 disease demonstrates abnormally shaped mitochondria and cytoplasmic glycogen beginning to accumulate between contractile elements $(\times 8500)$. (e) Satellite cells (red arrow) can be seen, by electron microscopy, beneath the basement membrane adjacent to damaged myocytes (black asterix; EM, $\times 10500$ ). (f) Al satellite cells, active and quiescent, express m-cadherin (red cell membrane staining, yellow arrows) but only activated satellite cells express myogenin (green nuclear staining, white arrow). A blue nuclear dye was used as a counterstain for all nuclei. (confocal microscopy, magnification, $\times 400$ ) . 
good short-term histologic response and also had a high proportion of type I fibers.

\section{Autopsy Findings from Patient B}

Skeletal myocytes of the deltoid and diaphragm examined at autopsy appeared as severely involved by glycogen accumulation (Figure $5 a$ and b) as the patient's antemortem quadriceps biopsies, consistent with the general motor and respiratory dysfunction seen clinically in this patient. The Schwann cells of small nerves embedded within the diaphragm also demonstrated moderate glycogen accumulation (Figure 5c). At autopsy, the heart weighed more than twice normal for age (115 vs $45 \mathrm{~g}$ ) and HRLM examination revealed considerable remaining lysosomal glycogen in the right and left ventricle as well as the interventricular septum (IVS) (Figure $5 \mathrm{~d})$. Electron microscopy of the IVS revealed multiple glycogen-containing vacuoles clustered in the center of each cardiomyocyte, some with ruptured vacuolar membranes and evidence of fusion with other vacuoles (Figure 5j). Smooth muscle cells of the muscularis propria of the bladder (Figure 5e) and the vascular media of a large artery (Figure $5 \mathrm{f}$ ) were also heavily infiltrated with abnormal accumulations of glycogen. CNS tissue samples revealed sporadic glycogen accumulation in Purkinje cells of the cerebellum (Figure 5g) and in cortical neurons (Figure 5h), however, the most dramatic glycogen accumulation was present in the motor neurons of the ventral horn in the spinal cord (Figure $5 \mathrm{i}$ and $5 \mathrm{k})$.

\section{Discussion}

The usage of digital histomorphometry to assess glycogen clearance proved to be a reasonable indicator of efficacy. These measurements demonstrated a clear relationship between glycogen clearance after 12 weeks of ERT and longer-term posttreatment outcome. This subset of individuals attained, then maintained, near normal glycogen levels for 52 weeks, demonstrating that rhGAA had been successfully targeted to skeletal myocytes and effectively re-enabled intralysosomal glycogenolysis. Lower baseline glycogen levels were associated with sustained clearance in patients $\mathrm{A}$ and $\mathrm{E}$ but not patient $\mathrm{F}$, suggesting that additional factors contribute to a positive long-term clinical outcome. When patients were examined with respect to age at baseline, it was notable that the two best histologic responders were also among the youngest patients at the onset of treatment (patients $A$ and $E$ were 4.7 and 3.1 months, respectively), however, other patients who began ERT around the same age showed essentially no histologic response (patient $\mathrm{B}$ began ERT at 4.8 months), suggesting that age alone is also not an independent determinant of outcome. Of note, genotype analysis performed on these patients did not allow any correlation analysis between genotype and disease severity at baseline, as most patients have a unique combination of GAA mutations. ${ }^{11}$ The characterization of GAA mutations, as well as CRIM status and antibody titers of these patients, are discussed in detail in a companion clinical publication. ${ }^{11}$

To further characterize the changes in pathology, we examined ultrastructure. By EM, baseline ultrastructural damage varied significantly among individual patient biopsies. In good histologic responders (patients $\mathrm{A}$ and $\mathrm{E}$ ) glycogen was predominantly membrane-bound, contained within cells in early stages 1 and 2 of disease progression. This glycogen should theoretically be accessible to exogenously delivered enzyme via receptormediated uptake from the cell surface to the lysosome. ${ }^{15}$ Furthermore, the catalytic activity of rhGAA is dependent on the acidic environment of the intact lysosome ${ }^{15}$ which would favor clearance of lysosomal glycogen over glycogen in the cytoplasm where the neutral $\mathrm{pH}$ would render the enzyme less active. The skeletal myocytes of good histologic responders also contained more intact mitochondria. As receptor uptake of the enzyme is an energy-dependent process, the integrity of mitochondria is likely an important factor in effective delivery of rhGAA to the lysosome.

The extent of ultrastructural damage in the myocytes from histologically non-responsive patient biopsies was more severe: contractile elements and mitochondria were scarce or absent in cells where glycogen predominated as non-membrane bound pools in the cytoplasm (Figure 4b and 4c). Variation in disposition of intracellular glycogen (membranebound vs cytoplasmic predominance) has been described previously by Griffin, who noted several progressive stages of ultrastructural change in skeletal myocytes from infantile Pompe disease. ${ }^{7}$ Griffin also proposed a 'lysosomal rupture hypothesis', asserting that during normal contraction, the unique contractile nature of myocytes subjects the glycogen-containing lysosomes to stress forces during the increased rigidity of surrounding myofibrils. Once the lysosomes reach a critical size, these forces cause lysosomal rupture and release of glycogen and lytic enzymes into the cytoplasm. The lytic enzymes cause damage to myofibrils, leading to loss of myofibrils and loss of contractile function. In more advanced stages (stages 4 and 5 in our grading system), myocytes filled with lakes of cytoplasmic glycogen and devoid of contractile elements were considered by Griffin and others to represent end stage disease. ${ }^{16-20}$ These changes are particularly relevant to ERT since this cytoplasmic glycogen released from lysosomes is probably inaccessible to the membrane receptor-dependent targeting mechanism. ${ }^{15}$ Even if delivery to the cytoplasm were possible, the neutral $\mathrm{pH}$ of the cytoplasm would render exogenously delivered rhGAA less active. 

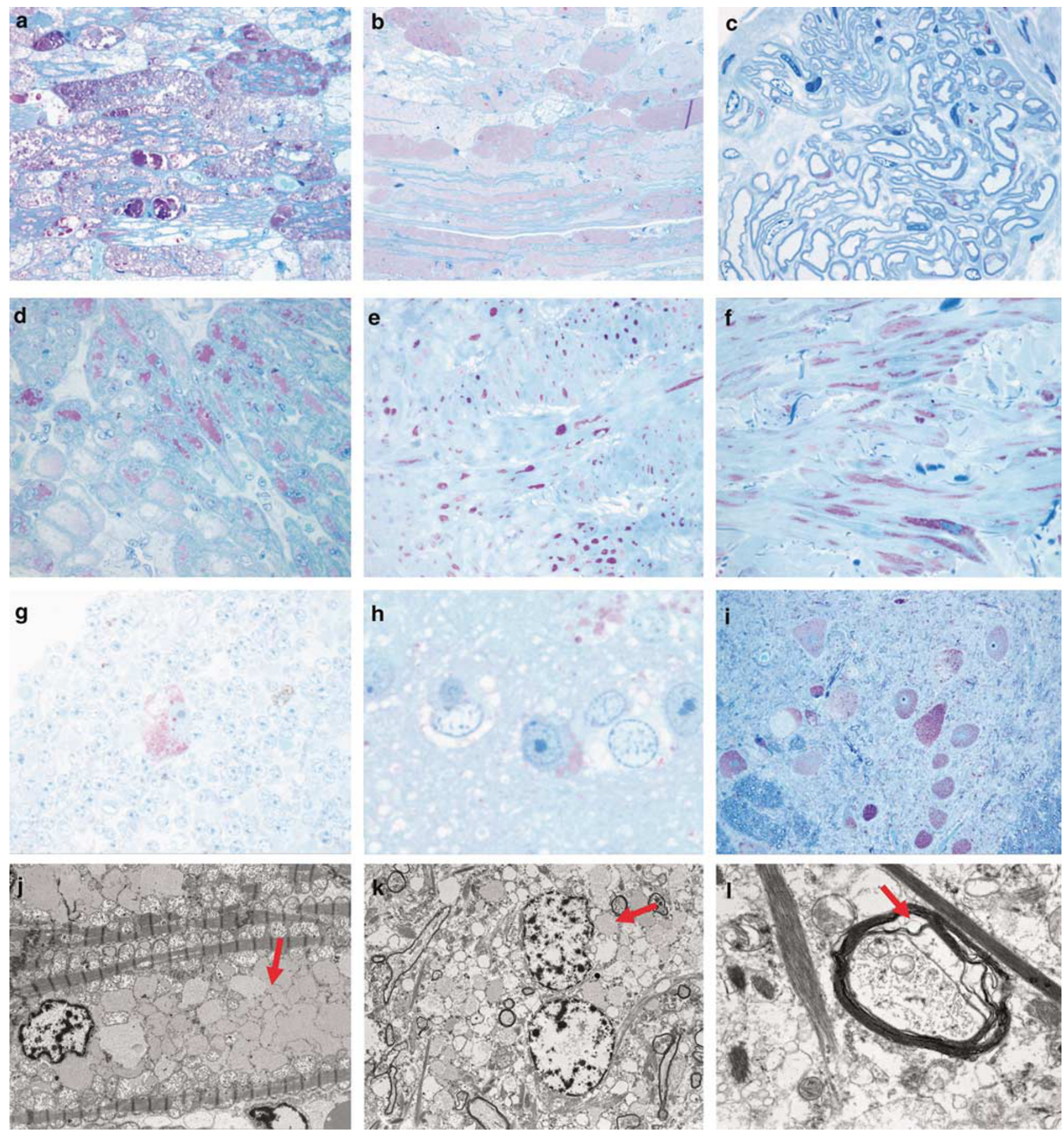

Figure 5 Examination of autopsy tissues from patient B demonstrates abnormal glycogen accumulation in multiple organs and cell types. (a) Skeletal muscle, deltoid (HRLM with Richardson's/ PAS stain, $\times 400$ ); (b) Skeletal muscle, diaphragm (HRLM with Richardson's/ PAS stain, $\times 400$ ); (c) Nerve from diaphragm: glycogen can be seen in Schwann cell cytoplasm (HRLM with Richardson's/ PAS stain, $\times 1000$ ); (d) Heart, interventricular septum (HRLM with Richardson's/ PAS stain, $\times 600$ ); (e) Bladder, smooth muscle cells of the muscularis propria (HRLM with Richardson's/ PAS stain, × 400); (f) Large artery, smooth muscle cells of the media (HRLM with Richardson's/ PAS stain, × 1000); (g) Cerebellum: glycogen accumulation can be seen in a Purkinje cell (HRLM with Richardson's/PAS stain, $\times 600)$; (h) Frontal lobe: glycogen accumulation in a neuron (HRLM with Richardson's/ PAS stain, × 1000). (i) Spinal cord: lysosomal glycogen can be seen in motor neurons of the ventral horn (HRLM with Richardson's/PAS stain, $\times 400$ ); (j) Cardiomyocyte of the interventricular septum contains multiple glycogen-filled lysosomes adjacent to the nucleus. (EM: $\times 28000)$. (k) Clusters of membrane-bound glycogen vacuoles are present in motor neurons of the ventral horn $(\mathrm{EM}$ : $\times 8800)$. (l) Myelin sheaths of nerve fibers exhibit splitting (EM: $\times 44000)$.

The concept that 'end-stage' cells such as those in Figure $4 \mathrm{~b}$ and c may be permanently unresponsive to current ERT regimens is supported by the absence of response in rare individual myocytes observed in patient biopsies exhibiting otherwise successful glycogen clearance (Figure 1b, cell with asterix). 
Similar to severely affected cells in poor histologic responders, these few remaining myocytes were completely filled with glycogen and devoid of contractile elements. Further, the existence of these nonresponsive cells in tissues from good histologic responders suggests that the proportion of end stage cells within a muscle may be a determinant of successful glycogen clearance. The individual cell staging and quantification we used to evaluate baseline biopsies confirmed that muscle from most poor histologic responders contained a high proportion of late stage myocytes with fewer early stage cells, a pattern incompatible with effective response to ERT and normal muscle function.

A surprising feature of this study was the absence of secondary pathology. Despite the severe intracellular pathology, there was no obvious evidence of cell necrosis or apoptosis by either light or electron microscopy. There was also no evidence of neutrophil, lymphocyte or macrophage infiltration to suggest recent or ongoing cell death, a finding consistent with observations by others. ${ }^{7}$ In addition, there were no areas of fibrosis to suggest remote cell death, yet all nonresponsive patients had elevated plasma levels of SGOT and CK, consistent with active, ongoing muscle insult. SGPT levels were also elevated. Elevation of muscle and liver enzymes has been noted by others ${ }^{21}$ and is consistent with glycogen accumulation observed at autopsy in muscle as well as in liver tissues. ${ }^{22-24}$ Furthermore, evidence of muscle regeneration was minimal. Abundant satellite cells were present in all posttreatment biopsies, however, only a fraction demonstrated signs of activation. Activation was elevated among the more severely affected, histologically unresponsive patients, and was relatively downregulated in the two best histologic responders. This suggested a regenerative response to ongoing injury in skeletal muscle with persistent glycogen accumulation. Satellite cell activation is dependent upon TGF- $\beta 2$ release from dying muscle fibers. ${ }^{25}$ In the absence of overt cell necrosis, such mechanisms of self-repair do not appear to be fully engaged in infantile Pompe muscle.

In light of the known differences in the glycolytic capacity of Type I (slow twitch) and Type II (fast twitch) muscle fibers, ${ }^{26}$ we have attempted to elucidate any potential impact of muscle fiber type on the response to ERT. Numerous studies have demonstrated higher capillary density among Type I fibers $^{9,10}$ as well as evidence of higher blood flow. ${ }^{27}$ Studies in mice also demonstrate a correlation between predominance of slow twitch fibers and more effective glycogen clearance ${ }^{28}$ while fast twitch fibers have been shown to have lower levels of lysosomal trafficking proteins and correspondingly less effective glycogen clearance. ${ }^{29}$ While exposure to rhGAA might be enhanced in muscle groups with greater bloodflow and capillary density, it is unclear from this small study whether the higher proportion of Type I fibers in the quadriceps of surviving patients $\mathrm{A}$ and $\mathrm{E}$ signaled an advantage. Additional biopsy data from ongoing trials are focused on further investigating this question. Interestingly, Type I fibers have also been associated with greater satellite cell content ${ }^{8,30,31}$ and hence, may have a greater capacity for repair.

Autopsy tissue samples from patient B, revealed multiple organ involvement, consistent with clinical reports on the nonskeletal manifestations of the disease. The cardiac hypertrophy and the prominence of remaining lysosomal glycogen in cardiomyocytes at autopsy was surprising, given the reported success in reducing LVMI (left ventricular mass index) scores and prolonging survival in these patients. ${ }^{11,32,33}$ Infiltration of septal myocytes has been reported in association with the shortened P-R intervals observed in some Pompe patients ${ }^{34}$ and a corrective increase in the P-R interval has been noted in conjunction with ERT. ${ }^{35}$ Involvement of vascular smooth muscle noted in the juvenile and adult forms of Pompe disease was reportedly responsible for vascular aneurysms resulting in severe headaches, cerebellar infarction and fatal rupture ${ }^{36}$ Glycogen accumulation in motor neurons of the spinal cord (Figure 5i) has also been reported previously in infantile patients ${ }^{18,37}$ and raises the question as to whether neuronal involvement is a secondary component contributing to muscle weakness. This area needs further investigation.

Pompe disease, like other lysosomal storage diseases (Gaucher, Fabry, Niemann-Pick) progresses over a lifetime, and clinical symptoms gradually worsen in the absence of treatment. However, in infantile Pompe disease, the clinical progression is rapid, resulting in a markedly narrow window of opportunity for interventional treatments. In addition, individual patient heterogeneity inevitably influences the rate of disease progression and further shortens the therapeutic window in patients with infantile Pompe disease who, when left untreated, often die before one year of age. The reason for higher doses of enzyme in Pompe disease compared to other lysosomal storage diseases (in the range of $1 \mathrm{mg} / \mathrm{kg}$ for Fabry and Gaucher disease) is at the moment unknown. Patients in this trial were dosed weekly with $10 \mathrm{mg} / \mathrm{kg}$; others studies have administered $20-40 \mathrm{mg} / \mathrm{kg}$ every other week. ${ }^{38} \mathrm{How}$ ever, investigators must balance any benefit of these higher doses with the increased risk of immune complex formation as illustrated in a case of reversible nephrotic syndrome. ${ }^{39}$ Additionally, the cardiac and respiratory involvement in this patient population puts them at an increased risk of arrhythmia and other significant medical complications during anesthesia and sedation procedures. ${ }^{40}$ Therefore, the decision to biopsy these patients should be approached with caution, and such decisions should be made judiciously and sparingly.

This study of baseline pathology and the histologic response of infantile Pompe patients to ERT highlights the severity and the heterogeneity of 
intracellular pathology that may account for the variability of clinical response. While age at onset of treatment may be one determinant, the extent and nature of baseline muscle pathology in individual patients may be additional determinants of response. The less robust histologic and clinical responses in patients who were older and/or presented with more advanced histologic disease, indicate that efforts to facilitate early peri-natal screening and diagnosis are warranted so that ERT can be provided when the disease is at its earliest, most responsive stage. The excellent histologic (and synchronous clinical) response to rhGAA therapy observed in two patients is very promising. At the time of this writing, patients $\mathrm{A}$ and $\mathrm{E}$ continue to walk and remain well on ERT. Current studies $^{41}$ are looking more closely at the effects that patient age and different dosing strategies have on histopathologic and clinical responses in order to optimize the successful treatment of this unique patient population.

\section{Acknowledgements}

We thank members of Genzyme's Department of Pathology who helped support this work, and Trent Richardson, our Medical Illustrator. We also thank the following clinical investigators and their patients who participated in this trial: A Amalfitano, YT Chen (DUMC, Durham); T Voit (University Hospital, Essen, Germany); M Nicolino (Pediatrique Hôpital Debrousse, Lyon, France); G Herman (Children's Hospital of Columbus); J Waterson (Children's Hospital of Oakland) RC Rogers (Greenwood Genetics Center); J Levine (Children's Hospital, Boston, MA). This work was funded by Genzyme Corporation. BLT, CL-M, CV, KA and MO'C are employees of Genzyme. PSK has received research/grant support and honoraria from Genzyme Corporation.

\section{References}

1 Hug G. Pre- and postnatal pathology, enzyme treatment, and unresolved issues in five lysosomal disorders. Pharmacol Rev 1979;90:565-591.

2 Phupong V, Shuangshoti S, Sutthiruangwong $\mathrm{P}$, et al. Prenatal diagnosis of Pompe disease by electron microscopy. Arch Gynecol Obstet 2005;271:259-261.

3 Hirschhorn R, Reuser AJJ. Glycogen storage disease type II: acid $\alpha$-glucosidase (acid maltase) deficiency. In: Scriver CR, Beaudet AL, Sly WS, Valle D (eds). The Metabolic and Molecular Basis of Inherited Disease, 8th edn. McGraw-Hill: New York, 2001, pp 3389-3420.

4 Van den Hout HM, Hop W, van Diggelen OP, et al. The natural course of infantile Pompe's disease: 20 original cases compared with 133 cases from the literature. Pediatrics 2003;112:332-340.

5 Kishnani PS, Hwu P, Mandel H, et al. A retrospective, multinational, multicenter study of the natural history of infantile Pompe disease. J Pediatr 2006;148: 671-676.

6 Lynch CM, Johnson J, Vaccaro C, et al. High resolution light microscopy (HRLM) and digital analysis of Pompe disease pathology. J Histochem Cytochem 2005;53:63-73.

7 Griffin JL. Infantile acid maltase deficiency. Virchows Arch 1984;45:23-36.

8 Hawke TJ, Garry DJ. Myogenic satellite cells: physiology to molecular biology. J Appl Physiol 2001;91: $534-551$.

9 Deveci D, Marshall JM, Egginton S. Relationship between capillary angiogenesis, fiber type, and fiber size in chronic systemic hypoxia. Am J Physiol Heart Circ Physiol 2001;281:H241-H252.

10 Porter MM, Stuart S, Boij M, et al. Capillary supply of the tibialis anterior muscle in young, healthy, and moderately active men and women. J Appl Physiol 2002;92:1451-1457.

11 Kishnani P, Nicolino M, Voit T, et al. Chinese hamster ovary cell-derived recombinant human acid alphaglucosidase in infantile-onset Pompe disease. J Pediatr 2006;149:89-97.

12 Piepenhagen PA, Nelson WJ. Defining E-cadherin associated complexes in epithelial cells: plakoglobin, beta and gamma catenin are distinct components. J Cell Sci 1993;104:751-762.

13 Bancroft JD, Stevens A. Theory and Practice of Histological Techniques, 3rd edn. Churchill Livingstone: New York, NY, 1990.

14 Sheehan DC, Sheehan BB. Theory and Practice of Histotechnology, 2nd edn. Batelle Press: Columbus, $\mathrm{OH}, 1980$.

15 Hesselink RP, Wagenmakers AJM, Drost MR, et al. Lysosomal dysfunction in muscle with special reference to glycogen storage disease type II. Biochem Biophys Acta 2003;1637:164-170.

16 Cardiff RD. A histochemical and electron microscopic study of skeletal muscle in a case of Pompe's disease (glycogenosis II). Pediatrics 1966;37:249-259.

17 Hug G, Garancis JC, Schubert WK, et al. Glycogen storage disease, type II, III, VIII, and IX. Am J Dis Child 1966;111:457-474.

18 Martin JJ, de Barsy T, Van Hoof F, et al. Pompe's disease: An inborn lysosomal disorder with storage of glycogen- A study of brain and striated muscle. Acta Neuropath 1973;23:229-244.

19 Garancis JC. Type II glycogenosis: biochemical and electron microscopic study. Am J Med 1968;44:289-300.

20 Hudgson P, Fulthorpe JJ. The pathology of type II skeletal muscle glycogenosis: a light and electronmicroscopic study. J Path 1975;116:139-147.

21 Iancu TC, Lerner A, Shiloh $\mathrm{H}$, et al. Juvenile acid maltase deficiency presenting as paravertebral pseudotumour. Eur J Pediatr 1988;147:372-376.

22 Temple JK, Dunn DW, Blitzer MG, et al. The 'muscular variant' of Pompe disease: clinical, biochemical and histologic characteristics. Am J Med Genet 1985;21:597-604.

23 Shotelersuk V, Shuangshoti S, Chotivitayatarakorn P, et al. Clinical, pathological and electron microscopic findings in two Thai children with Pompe disease. J Med Assoc Thai 2002;85(Suppl 1):S271-S279.

24 Teng YT, Su WJ, Hou JW, et al. Infantile-onset glycogen storage disease type II (Pompe disease): report of a case with genetic diagnosis and pathological findings. Chang Gung Med J 2004;27:379-384. 
25 McLennan I, Koishi K. Cellular localization of transforming growth factor-beta2 and -beta3 (TGF- $\beta 2$, TGF- $\beta 3$ ) in damaged and regenerating skeletal muscles. Dev Dyn 1997;208:278-289.

26 Herbison GJ, Jaweed MM, Ditunno JF. Muscle fiber types. Arch Phys Med Rehabil 1982;63:227-230.

27 Terjung RL, Engbretson BM. Blood flow to different rat skeletal muscle fiber type sections during isometric contractions in situ. Med Sci Sports Exerc 1988; 20:S124-S130.

28 Raben N, Danon M, Gilbert AL, et al. Enzyme replacement therapy in the mouse model of Pompe disease. Mol Genet Metab 2003;80:159-169.

29 Fukada T, Ewan L, Bauer M, et al. Dysfunction of endocytic and autophagic pathways in a lysosomal storage disease. Ann Neurol 2006;59:700-708.

30 Schmalbruch H, Hellhammer U. The number of nuclei in adult rat muscles with special reference to satellite cells. Anat Record 1977;189:169-176.

31 Gibson MA, Schultz E. The distribution of satellite cells and their relationship to specific fiber types in soleus and extensor digitorum longus muscles. Anat Record 1982;202:329-337.

32 Amalfitano A, Bengur AR, Morse RP, et al. Recombinant human acid alpha-glucosidase enzyme replacement therapy for infantile glycogen storage disease type II: results of a phase I/II clinical trial. Genet Med 2001;3:132-138.

33 Van den Hout JM, Kamphoven J, Winkel L, et al. Long-term intravenous treatment of Pompe disease with recombinant human $\alpha$-glucosidase from milk. Pediatrics 2004;113:e448-e456.

34 Bharati S, Serratto M, DuBrow I, et al. The conduction system in Pompe's disease. Pediatr Cardiol 1982;2: 25-32.

35 Ansong A, Li JS, Nozik-Grayck E, et al. Electrocardiographic response to enzyme replacement therapy for Pompe disease. Genet Med 2006;8:297-301.

36 Makos MM, McComb RD, Hart MN, et al. Alphaglucosidase deficiency and basilar artery aneurysm: report of a sibship. Ann Neurol 1987;22:629-633.

37 Martini C, Ciana G, Benettoni A, et al. Intractable fever and cortical neuronal glycogen storage in glycogenosis type 2. Neurology 2001;57:906-908.

38 Cook AL, Kishnani PS, Carboni MP, et al. Ambulatory electrocardiogram analysis in infants treated with recombinant human acid $\alpha$-glucosidase enzyme replacement therapy for Pompe disease. Genet Med 2006;8:313-317.

39 Hunley TE, Corzo D, Dudek M, et al. Nephrotic syndrome complicating alpha-glucosidase replacement therapy for Pompe disease. Pediatrics 2004;144:e532-e535.

40 Ing RJ, Cook DR, Bengur RA, et al. Anaesthesia management of infants with glycogen storage disease type II: a physiological approach. Paediatr Anaesth 2004;14:514-519.

41 Kishnani PS, Corzo D, Nicolino M, et al. Recombinant Human Acid $\alpha$-glucosidase: major clinical benefits in infantile onset Pompe disease. Neurology 2006 (in press).

Supplementary Information accompanies the paper on the Laboratory Investigation website (http:// www.nature.com/labinvest) 